at the expense of integrity and availability, and regulations/ instructions are used as an excuse not to change even vital information. The mental-picture of cyber security should turn from "threat, crime, attack" to "trust". Creating confidence in safe digital future is truly needed in the integration of the digital and physical world's leading to a new digital revolution. The precondition for the exchange of information "trust" must be systematically built at every CPS' level (platform, software, people).

\section{ASSESSMENT OF HOSPITAL QUALITY AND HEALTH CARE PROVIDERS PERFORMANCES DURING MNH CARE IN BANGLADESH}

Farzana Islam, Aminur Rahman, Abdul Halim, AKM Fazlur Rahman. Centre for Injury Prevention and Research, Bangladesh (CIPRB)

\subsection{6/injuryprev-2016-042156.779}

Background Despite the progress towards the MDG 4 and 5, compared to the developed world maternal and newborn deaths are still high in Bangladesh and poor quality of maternal and newborn health care is often blamed for this high mortality. This study assessed two important components of quality of maternal and newborn health care namely, infrastructure and performance of health care providers of public hospitals in Bangladesh.

Methods Cross sectional survey was conducted to measure the quality of infrastructures and assess the clinical care performance of the health care providers related to maternal and newborn services in 14 district and sub-district level public hospitals of Thakurgaon and Jamalpur districts. Six components including human resource, physical infrastructure, infection prevention, equipments/logistics/supplies, essential drugs and recordkeeping were assessed under infrastructure. Maternal and newborn care services provided by the health care providers were evaluated during antenatal, postnatal, delivery and newborn care. The study was conducted from November to December 2011. The average of the sub-items of each item was calculated and then the mean average of the items were estimated.

Results The percentage of mean average scores of all items of infrastructure for district hospitals, maternal and child welfare centres and upazila health complexes were 57.1\%, 52.7\% and $45.9 \%$ respectively, which were below the cut-off point $(60.0 \%)$. None of the health care providers of three types of government hospitals obtained $100 \%$ score in any areas namely antenatal care, post-natal care, delivery care and newborn care.

Conclusions Quality of infrastructure of health facilities, and clinical care performance of the health care providers during discharging maternal and newborn health services were found poor in district and sub-district level public hospitals of Bangladesh.

\section{Trauma Care and Rehabilitation}

\section{Post Tue 2.19}

\section{ASSOCIATION BETWEEN APACHE II SCORES, TRISS SCORES, AND THE OUTCOME OF CARE IN TRAUMA PATIETNS}

\footnotetext{
${ }^{1}$ Supaporn Tansura, ${ }^{2}$ Donwiwat Saensom, ${ }^{3}$ Pothipong Ruengjui. ${ }^{1}$ Trauma Surgical ICU, Khon Kaen Hospital, Thailand; 'Faculty of Nursing, KhonKaen University, Thailand; ${ }^{3}$ Department of Surgery, Khon Kaen Hospital, Thailand
}

Background In recent years, several trauma scoring systems have been developed and validated for use in prediction of outcome. TRISS combines physiologic and anatomic components of injury with age to characterise the severity of injury. The APACHE II score consists of 12 physiologic variables, pre morbid health ,and patient age . To investigate the association between APACHE II score, TRISS score and the outcome of care among trauma patients.

Methods The retrospective descriptive study was undertaken during 2012-2014. Medical records of 209 patients admitted to the Trauma ICU were reviewed for age, sex, admitting APACHE II and TRISS scores, length of ICU stay and outcome of care. Outcome of care referred to whether the patients died or survived at discharge. Multiple logistic regression was used to construct the prediction model.

Results Factors significantly and independently associated with the outcome of Trauma ICU care were APACHE II score, TRISS score, and length of ICU stay. For each point increased of APACHE II score, patient survival rate decreased 1.12 times (Adjusted odds ratio: $\mathrm{AOR}=1.12,95 \% \mathrm{CI}:=1.04-1.22$ ). However, when ICU length of stay increased 1 day survival rate increased 1.26 times $(\mathrm{AOD}=1.26,95 \% \mathrm{CI}:=1.12-1.41)$. Similarly, when TRISS score increased 0.1 point, survival rate increased 5.42 times (AOD = 5.52-532.67).

Conclusions Less severe illness (lower APACHE II score), higher probability of survival (higher TRISS score) and longer ICU length of stay significantly predicted patient survival in the Trauma ICU.

\section{NUTRITIONAL STATUS OF CRITICALLY ILL PATIENTS AND THE OUTCOME OF CARE}

'Supaporn Tansura, ${ }^{2}$ Donwiwat Saensom, 'Apiwan Insee. 'Trauma Surgical ICU, Khon Kaen Hospital, Thailand; ${ }^{2}$ Faculty of Nursing, Khon Kaen University, Thailand

\subsection{6/injuryprev-2016-042156.781}

Background Malnutrition is one of the most common problems found in critically ill patients. It is often associated with increased mortality rate and other complications in an intensive care unit (ICU). Previous studies indicated that $40 \%-50 \%$ of critically ill patients are malnourished and an average of 5 kilogram body weight is lost when they are discharged.

Methods This prospective study was conducted to survey the nutritional status of critically ill patients in the Trauma surgical Intensive Care Unit (ICU Trauma) in Khon Kaen Hospital, Thailand, and identify potential outcomes of malnutrition among these patients. From October 2012 to July 2013, 282 patients were screened with the Nutrition Alert Form (NAF) in the second day of their admission. Higher NAF scores signify poorer nutritional status. The patients were then followed until they were discharged from ICU Trauma.

Results Revealed that, 2 days after admission, over a half of the patients $(\mathrm{n}=180,63.8 \%)$ were withheld from oral intake of food and fluids. Moreover, most patients were severely malnourished ( $\mathrm{n}=240,85.1 \%)$. When considering the success of endotracheal tube (ETT) removal as an outcome of care, it was found that those with successful ETT removal had significantly lower NAF score $(=13.61$, S.D. $=4.4)$ compared to those who failed ETT extubation $(=17.08$, S.D. $=9.7, \mathrm{t}=-3.345, \mathrm{p}=0.001$ ). NAF scores were not different between patients who survived and those who died in this study. These results indicated that critically ill patients are at high risk for malnutrition. Proper 